\title{
Study of Industrial Black Water Treatment Plant Installation Planning System Based on Sewage Treatment Plant
}

\author{
Jantiara Eka Nandiasa dan Ridi Nurardiansyah \\ Faculty of Engineering, University Mercu Buana, Jakarta, Indonesia \\ jantiara@mercubuana.ac.id, ridinura24@gmail.com
}

\begin{abstract}
Wastewater in general is water, in general, is a liquid that comes from households, industries, or other public places that usually contain materials or substances that can endanger human life and disturb the environment. Quid comes from households, industries, or other public places that usually contain materials or substances that can endanger human life and disturb the environment. This study to plan, analyze, and assess the performance conditions of wastewater treatment plants (IPAL) at PT XYZ Bekasi Regency on environmental quality standards. Data and information used are data on the amount of wastewater, sources of wastewater, and behavior of water use as well as secondary data in the form of data that the authors surveyed directly at the research location. The processing method of the output value of WWTP uses the research method of the laboratories from the research method in which the output value exceeds the quality standard of LHK Regulation No. 68 the Year 2016 and has exceeded its planning capacity. And what is identified is not safe. An alternative solution to the problem of unsafe output values is by planning a new wastewater treatment plant.
\end{abstract}

\section{Keywords}

Waste Water Treatment Plant, Black Water, Sewage Treatment Plant

\section{Introduction}

Wastewater or waste water is residual water that is discharged from households, industries and other public places, and in general contains materials or substances that can be harmful to human health and disturb the environment. Another limitation says that wastewater is a combination of liquid and liquid waste that comes from residential, commercial, office and industrial areas together with ground water, surface water, and rainwater that may be present. (Notoatmodjo, 2003).

Bekasi Regency as an industrial city shows serious symptoms regarding environmental problems originating from industry and housing activities. Every day industrial discharges which show an increase based on the correlation of the growth of factories in the Bekasi district this condition can damage the environment resulting in a decrease in the level of health of the population around the industry.

At PT XYZ in Bekasi district is a company engaged in the manufacturing sector with an actual human resource of 279 in the January 2020 data value of the output at the wastewater treatment plant exceeds the quality standard of LHK candy no 68 in 2016, while the number of projected data sources maximum human power of 315 people.

Based on the explanation above, the problem in this final project is the incomplete value of the output of the existing wastewater treatment plant according to the LHK Permen quality standard No. 68 year 2016 with the condition that the amount of human resources has not been maximally projected.

\section{Methodology}

The type of research used is analyzing by observing the laboratory method with the aim to determine the effectiveness of Waste Water Treatment Plant (WTP) based on Industrial WTP in Industry and for planning a Waste Water Treatment Plant based Sewage Treatment Plant (STP) in Industry. 


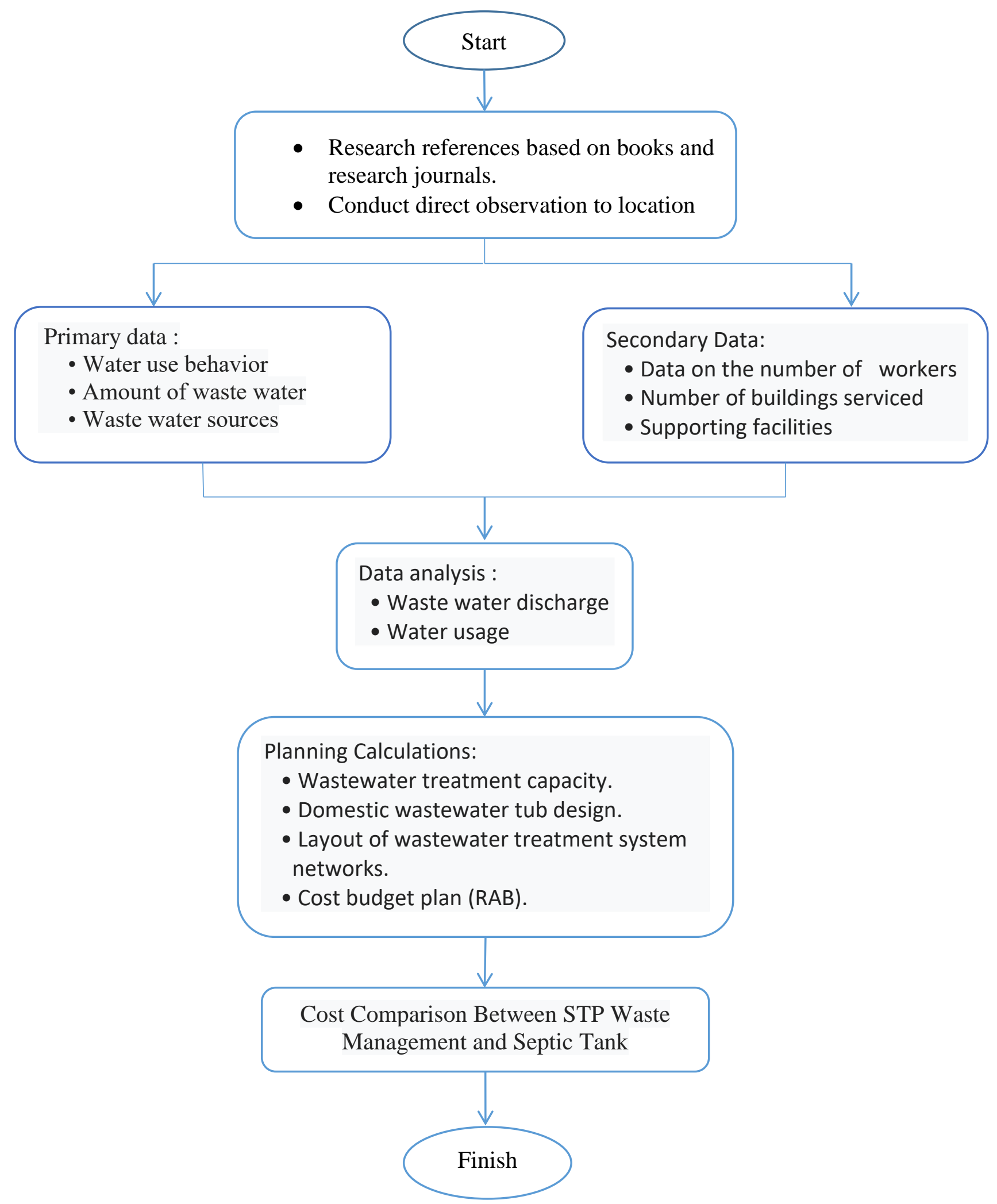

Picture 1 Flowchart of research procedure 


\subsection{Research Location and Time}

The research location is at PT XYZ MM2100 industrial area, West Cikarang District, Bekasi Regency. And the time of the study was conducted in September 2019 - January 2020.

\subsection{Study Literatur}

Literature or reference materials used in this thesis include:

- Metcalg and Eddy, 2003, Wastewater Engineering Treatment and Reuse, McGraw

- Suharto, 2011, Limbah Kimia Dalam Pencemaran Udara dan Air, Andi Yogyakarta, Yogyakarta

- Kusnoputranto, Haryoto, 1985, Kesehatan Lingkungan, FKM UI, Jakarta

- Tchobanoglous, G, 1985, Teknik Sumber Daya Air, Terjemahan oleh Djoko Sasongko, 1991, Erlangga, Jakarta

\subsection{Research Location Survey}

a) Primary data :

Sources of liquid waste

Liquid waste discharge

Water use behavior

\section{b) Sekuder data}

Data on the number of workers

Number of buildings served

Supporting facilities

\subsection{Data analysis}

The collected data is then analyzed descriptively as follows:

Planning a domestic wastewater treatment system

Calculate the discharge of wastewater per day / m3

\subsection{Domestic Wastewater Treatment System Design}

Wastewater tub design

Layout of wastewater treatment system networks

Cost budget plan (RAB)

\subsection{Cost Comparison Between Sewage Treatment Plant and Septic Tank Waste Management}

Waste water treatment plant construction

Area of land used

Construction time

Total cost

\subsection{Conclusions and recommendations}

\section{Result and Analysis}

\subsection{Water use behavior}

The survey was conducted to find out the behavior of water in daily life which will be input to the wastewater treatment plant in the table below: 
Table 1. Water use behavior

\begin{tabular}{|c|c|}
\hline Time & Use \\
\hline $0.00-1.00$ & Empty \\
\hline $1.00-2.00$ & Empty \\
\hline $2.00-3.00$ & Empty \\
\hline $3.00-4.00$ & Prayer \\
\hline $5.00-6.00$ & Prayer \\
\hline $6.00-7.00$ & Toilet \\
\hline $7.00-8.00$ & Empty \\
\hline $8.00-9.00$ & Toilet \\
\hline $9.00-10.00$ & Empty \\
\hline $10.00-11.00$ & Break \\
\hline $11.00-12.00$ & Empty \\
\hline $12.00-13.00$ & Break + Prayer \\
\hline $13.00-14.00$ & Prayer \\
\hline $14.00-15.00$ & Toilet \\
\hline $15: 00-16: 00$ & Prayer \\
\hline $16.00-17.00$ & Prayer \\
\hline $17.00-18.00$ & Break + Prayer \\
\hline $18.00-19.00$ & Break + Prayer \\
\hline $19.00-20.00$ & Prayer \\
\hline $20.00-21.00$ & Prayer \\
\hline $21.00-22.00$ & Empty \\
\hline $22.00-23.00$ & Toilet \\
\hline $23.00-24.00$ & Empty \\
\hline
\end{tabular}

Source: Survey results

\subsection{Source of Wastewater and Amount of Wastewater}

The mapping of wastewater sources at PT XYZ in Bekasi Regency aims to find out what inputs will be input at the wastewater treatment plant, in the table below:

Table 2. Waste Water Source

\begin{tabular}{|c|c|c|c|c|}
\hline NO & AREA & WASTE WATER SOURCE & AMOUNT & INFORMATION \\
\hline 1 & \multirow{4}{*}{ Workshop } & WC & 10 & Active \\
\hline 2 & & Urine & 7 & Active \\
\hline 3 & & Floor Drain & 11 & Active \\
\hline 4 & & Washtaple & 6 & Active \\
\hline 5 & \multirow{4}{*}{ Production Proses } & WC & 5 & Active \\
\hline 6 & & Urine & 5 & Active \\
\hline 7 & & Floor Drain & 7 & Active \\
\hline 8 & & Washtaple & 5 & Active \\
\hline
\end{tabular}




\begin{tabular}{|c|c|c|c|c|}
\hline 9 & \multirow{4}{*}{ Production Fillpack } & WC & 6 & Active \\
\hline 10 & & Urine & 3 & Active \\
\hline 11 & & Floor Drain & 8 & Active \\
\hline 12 & & Washtaple & 6 & Active \\
\hline 13 & \multirow{4}{*}{ Mushola } & $\mathrm{WC}$ & 2 & Active \\
\hline 14 & & Urine & - & Active \\
\hline 15 & & Floor Drain & 1 & Active \\
\hline 16 & & Washtaple & 2 & Active \\
\hline 17 & \multirow{4}{*}{ Loker } & WC & 3 & Active \\
\hline 18 & & Urine & 2 & Active \\
\hline 19 & & Floor Drain & 3 & Active \\
\hline 20 & & Washtaple & 5 & Active \\
\hline 21 & Cateen & Washtaple Cleaning Area & 3 & Active \\
\hline 22 & Loker & Washtaple Pantry & 1 & Active \\
\hline
\end{tabular}

\subsection{Number of Workers Data}

a. Number of Actual Workers

Data on the number of human resources as a reference to determine the dimensions of the body that will be planned at the waste water treatment plant (IPAL) based on Sewage Treatment Plant in the table below:

Table 3. Number of actual workers

\begin{tabular}{|l|c|c|}
\hline \multicolumn{1}{|c|}{ JAM KERJA } & JUMLAH PEGAWAI (orang) & AIR LIMBAH DIHASILKAN (m3/hari) \\
\hline Produksi Fillpack & 214 & 17,1 \\
\hline Produksi Proses & 47 & 3,76 \\
\hline Enginering & 18 & 1,44 \\
\hline \multicolumn{2}{|c|}{ Minimum } & 1,44 \\
\hline \multicolumn{2}{|c|}{ Maksimal } & 17,1 \\
\hline \multicolumn{2}{|c|}{ Debit per day } & 22,3 \\
\hline
\end{tabular}

Source: PT XYZ work schedule

\section{b. Maximum Projection Number of Workers Data}

Data on the number of human resources as a reference to determine the dimensions of the body that will be planned at the waste water treatment plant (IPAL) based on Sewage Treatment Plant in the table below: 
Table 4. Maximum projection number of wokers data

\begin{tabular}{|l|c|c|}
\hline \multicolumn{1}{|c|}{$\begin{array}{c}\text { WORKING } \\
\text { HOURS }\end{array}$} & $\begin{array}{l}\text { NUMBER OF EMPLOYEES } \\
\text { (PEOPLE) }\end{array}$ & WASTE WATER PRODUCED (m3/day) \\
\hline Shift 1 & 120 & 9,6 \\
\hline Non Shift & 60 & 4,8 \\
\hline Shift 2 & 100 & 8 \\
\hline Shift 3 & 35 & 2,8 \\
\hline \multicolumn{2}{|c|}{ Minimum } & 2,8 \\
\hline \multicolumn{2}{|c|}{ Maksimal } & 9,6 \\
\hline & Debit per day & 25.2 \\
\hline
\end{tabular}

Source: Human Resource department projection data

The capacity of domestic wastewater produced by PT XYZ, as a reference to determine the dimensions of the wastewater treatment tank to be planned. Wastewater produced is related to clean water that is used for daily needs.

$$
\begin{array}{ll}
\text { Total human resources } & =315 \text { person } \\
\text { Liquid waste } & =80 \text { liter/person/day } \\
\text { Total liquid waste } & =\text { Total SDM } \times \text { liquid waste per person } \\
& =315 \text { person } \times 80 \text { liter/person } / \text { day } \\
& =25.200 \text { liter } / \text { day } \rightarrow 25,2 \mathrm{~m}^{3} / \text { day }
\end{array}
$$

Total waste water treatment capacity $=25.200$ liter $+(15 \% \times 25.200$ liter $)$

$$
=30.000 \text { liter/day } \rightarrow 30 \mathrm{~m}^{3} / \text { day }
$$

\subsection{Calculation of Waste Water Treatment Plant Installation Planning Based on Sewage Treatment Plant}

\subsubsection{Screening}

Wastewater discharge $(\mathrm{Q})=30 \mathrm{~m} 3 / \mathrm{day}$

BOD in $\quad=350 \mathrm{mg} / \mathrm{l}$

BOD ef $\quad=315 \mathrm{mg} / \mathrm{l}$

Efficiency $\quad=10 \%$

Hours running time $\quad=1,5 \mathrm{jam}$

Tub volume required

$$
\begin{aligned}
& =\mathrm{Q} \text { in } \times \text { HRT } \\
& =\frac{1,5 \mathrm{~h}}{24 \mathrm{~h}} \times 30 \mathrm{~m}^{3} / \text { day } \\
& =1,875 \mathrm{~m}^{3} / \text { day }
\end{aligned}
$$

After knowing the volume value, the dimensions can be determined according to the volume as follows:

$$
\begin{aligned}
& \text { High }=1,45 \mathrm{~m} \\
& \text { Long }=0,7 \mathrm{~m} \\
& \text { Wide }=1,8 \mathrm{~m} \\
& \text { Freeboard }=0,3 \mathrm{~m} \\
& \text { Check HRT averge : } \\
& \mathrm{T}=\frac{\mathrm{P} \times \mathrm{L} \times \mathrm{T}}{\mathrm{Q}} \times 24 \mathrm{~h} / \text { day } \\
& \mathrm{T}=\frac{0,7 \mathrm{~m} \times 1,8 \mathrm{~m} \times 1,45 \mathrm{~m}}{30 \mathrm{~m}^{3} / \text { day }} \times 24 \mathrm{~h} / \text { day } \\
& \mathrm{T}=1,4616 \text { hours } \\
& \text { Check Surface Loading : } \\
& \text { Surface loading }=\frac{\mathrm{Q}}{\mathrm{L} \times \mathrm{P}}
\end{aligned}
$$


Surface loading $=\frac{30 \mathrm{~m}^{3} / \text { day }}{1,8 \mathrm{~m} \times 0,7 \mathrm{~m}}=23,8 \mathrm{~m}^{3} /$ day

Surface loading $20-50 \mathrm{~m}^{3} / \mathrm{m}^{2}$. hari (JWWA)

3.4.2 Biofilter Anaerob

$\mathrm{Q}=30 \mathrm{~m}^{3} /$ day

BOD in $\quad=315 \mathrm{mg} / \mathrm{l} \rightarrow 0,315 \mathrm{~kg} / \mathrm{m}^{3}$

BOD ef $\quad=126 \mathrm{mg} / \mathrm{l} \rightarrow 0,126 \mathrm{~kg} / \mathrm{m}^{3}$

Efficiency $=40 \%$

For water treatment using a standard biofilter process, the BOD load per volume of media $0,4-4,7 \mathrm{~kg}$ $\mathrm{BOD} / \mathrm{m}^{3}$. day

Determined the BOD load used : 4,7 kg $\frac{\text { BOD }}{\mathrm{m}^{3}}$. day

BOD load in waste water

BOD load $=Q \times$ BOD in

BOD load $=30 \mathrm{~m}^{3} /$ day $\times 0,315 \mathrm{~kg} / \mathrm{m}^{3}$

BOD load $=9,45 \mathrm{~kg} /$ day

Media voluume required

$\mathrm{V}=\frac{\text { BOD load }}{\text { Standart BOD load }}$

$\mathrm{V}=\frac{9,45 \mathrm{~kg} / \text { day }}{4,7 \frac{\mathrm{kg}}{\mathrm{m}^{3}} \cdot d a y}=2 \mathrm{~m}^{3}$

Volume media $=40 \%$ of the total reactor volume

Reactor volume required

$\mathrm{V}=\frac{100}{\mathrm{~V} \text { media reaktor }} \times \mathrm{V}$ media

$\mathrm{V}=\frac{100}{40} \times 2 \mathrm{~m}^{3}=5 \mathrm{~m}^{3}$

The residence time in the Anerob reactor

$\mathrm{T}=\frac{\mathrm{V} \text { reaktor }}{\mathrm{Q}}$

$\mathrm{T}=\frac{5 \mathrm{~m}^{3}}{1,25 \mathrm{~kg} / \text { day }}=4$ hours

After knowing the volume value, the dimensions can be determined according to the volume as follows:

$\begin{array}{lc}\text { Effective depth } & =1,45 \mathrm{~m} \\ \text { Media high } & =0,6 \mathrm{~m} \\ \text { High space above the media } & 0,3 \mathrm{~m} \\ \text { High space sludge } & =0,3 \mathrm{~m} \\ \text { Long } & =2 \mathrm{~m} \\ \text { Wide } & =1,75 \mathrm{~m} \\ \text { Freeboard } & =0,3 \mathrm{~m} \\ \text { Effective volueme } & =5 \mathrm{~m}^{3}\end{array}$

Check the residence time in the Anaerobic reactor:

$\mathrm{T}=\frac{\mathrm{V} \text { reaktor }}{\mathrm{Q}} \times 24 \mathrm{~h} / \mathrm{d}$

$\mathrm{T}=\frac{5 \mathrm{~m}^{3}}{30 \mathrm{~m}^{3} / \text { day }} \times 24 \mathrm{~h} / \mathrm{d}=4$ hours

3.4.4 Bio Filter Aerob

$\begin{array}{ll}\mathrm{Q} & =30 \mathrm{~m} 3 / \text { day } \\ \text { BOD in } & =126 \mathrm{mg} / \mathrm{l} \rightarrow 0,126 \mathrm{~kg} / \mathrm{m}^{3} \\ \text { BOD ef } & =50,4 \mathrm{mg} / 1 \rightarrow 0,0504 \mathrm{~kg} / \mathrm{m}^{3} \\ \text { Efficiency } & =40 \% \\ \text { BOD load in waste water }\end{array}$


BOD load $=Q \times$ BOD in

BOD load $=30 \mathrm{~m}^{3} /$ hari $\times 0,126 \mathrm{~kg} / \mathrm{m}^{3}$

BOD load $=3,78 \mathrm{~kg} /$ day

The amount of BOD removed

BOD removed $=\frac{\text { Efficiency }}{100} \times$ BOD load

BOD removed $=\frac{40}{100} \times 3,78 \mathrm{~kg} /$ day

BOD removed $=1,51 \mathrm{~kg} /$ day

BOD load per media volume $=4 \mathrm{~kg}$ BOD $/ \mathrm{m} 3$.day

Media volume required

$\mathrm{V}$ media $=\frac{\text { BOD load waste water }}{\text { BOD load per Volume }}$

$\mathrm{V}$ media $=\frac{3,78 \mathrm{~kg} / \text { day }}{4 \mathrm{~kg} / \mathrm{m}^{3} \text { day }}=0,94 \mathrm{~m}^{3}$

Volume media $=40 \%$ from the reactor volume

Volume reaktor $=\frac{100}{40} \times \mathrm{V}$ media

Volume reaktor $=\frac{100}{40} \times 0,94 \mathrm{~m}^{3}=2,35 \mathrm{~m}^{3}$

Time of residence required by HRT

$\mathrm{T}=\frac{\mathrm{V} \text { reaktor }}{\mathrm{Q}}$

$\mathrm{T}=\frac{2,35 \mathrm{~m}^{3}}{1,25 \mathrm{~m}^{3} / \text { hours }}=1,88$ hours

After knowing the volume value, the dimensions can be determined according to the volume as follows:

$\begin{array}{ll}\text { Effective depth } & =1,45 \mathrm{~m} \\ \text { Media high } & =0,58 \mathrm{~m}\end{array}$

High space above the media $=0,3 \mathrm{~m}$

High space sludge $\quad=0,57 \mathrm{~m}$

Long $\quad=0,93 \mathrm{~m}$

Wide $\quad=1,75 \mathrm{~m}$

Freeboard $\quad=0,3 \mathrm{~m}$

Check the residence time in the Aerobic reactor:

$\mathrm{T}=\frac{\mathrm{V} \text { reaktor }}{\mathrm{Q}} \times 24 \mathrm{~h} /$ day

$\mathrm{T}=\frac{2,35 \mathrm{~m}^{3}}{30 \mathrm{~m}^{3} / \text { day }} \times 24 \frac{\text { hours }}{\text { day }}=1,88$ hours

3.4.5 Final settling tub

$\mathrm{Q} \quad=32,8 \mathrm{~m} 3 / \mathrm{day}$

BOD in $\quad=50,4 \mathrm{mg} / \mathrm{l} \rightarrow 0,05 \mathrm{~kg} / \mathrm{m}^{3}$

BOD ef $\quad=47,88 \mathrm{mg} / \mathrm{l} \rightarrow 0,0478 \mathrm{~kg} / \mathrm{m}^{3}$

Efficiency $=5 \%$

Hours running time 1,5 hours -2 hours, and HRT used 1,5 hours

Tub volume required

$\mathrm{V}=\frac{\mathrm{HRT}}{24 \mathrm{~h}} \times \mathrm{Q}$

$\mathrm{V}=\frac{1,5 \mathrm{~h}}{24 \mathrm{~h}} \times 1,366 \mathrm{~m}^{3}=$

After knowing the volume value, the dimensions can be determined according to the volume as follows:

Wide

$$
=1,8 \mathrm{~m}
$$




$$
\begin{aligned}
& \text { Effective depth }=1,45 \mathrm{~m} \\
& \text { Long }=0,8 \mathrm{~m} \\
& \text { Freeboard }=0,3 \mathrm{~m} \\
& \text { 3.4.6 Budget Plan (RAB) }
\end{aligned}
$$

Development costs in waste water treatment plant (WWTP) based on Sewage Treatment Plant (STP) in the table below

Table 5. Budget plan

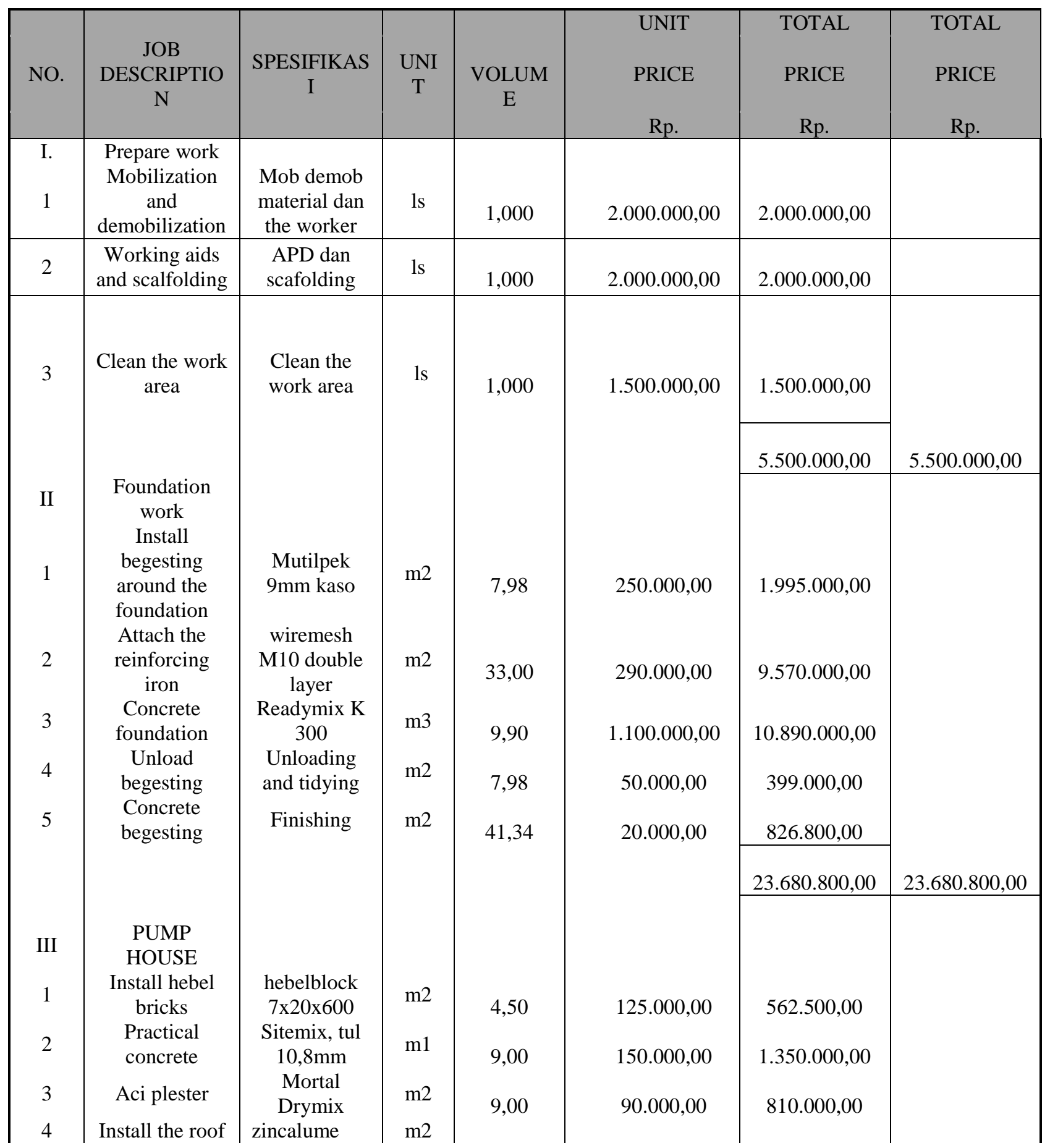




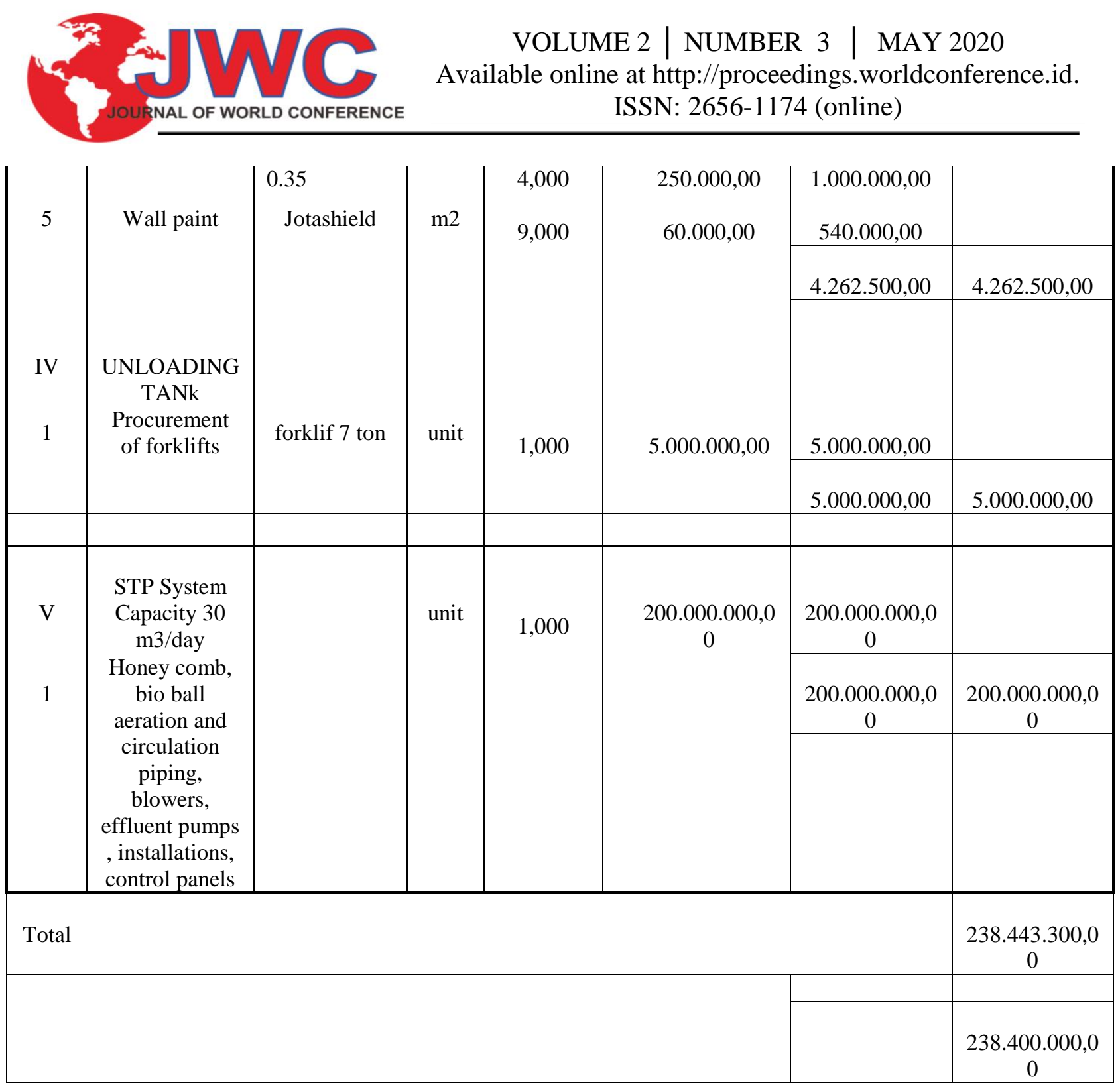

\section{Conclussion}

From the results of the field survey and analysis the conclusions obtained are:

1. Domestic wastewater discharge generated by PT XYZ with projected human resource data is a maximum of $25.5 \mathrm{~m}^{\wedge} 3$ / day or 25,500 liters / day.

2. The domestic wastewater treatment system used is a centralized system

3. From the results of the calculation can be the size of the Wastewater Treatment Plant (IPAL) based on Sewage Treatment Plant (STP) that is $=2000 \mathrm{~mm} \times 6500 \mathrm{~mm}$

4. This Wastewater Treatment Plant (IPAL) serves 315 employees.

5. The planning process of this Waste Water Treatment Plant (WWTP) amounts to Rp. $238,443,300.00$.

\section{References}

Basics Of Waste Water Management. Sugiharto. 1987. Jakarta : Universty Indonesia, 1987.

Chemical Waste In Air And Water Pollution. Suharto. 2011. Yogyakarta : Andi Yogyakarta, 2011.

Environmental Health. Kusnoputranto, Haryoto. 1985. Jakarta : University Indonesia, 1985.

Environmental Protection Agency. Willgooso. 1975. 1975.

Evalution Of Community Based Communal Waste Water Treatment Plant In Panukang District. Ali, Muhammad. 2015. Makassar : s.n., 2015.

Fardiaz, Srikandi. 1992. Water And Air Populations. Yogyakarta : Canisius, 1992. 
Hadi, A. 2005. Principles Of Management Of Environmental Sampling. Jakarta : Gramedia Pustaka Utama, 2005. Waste Water Engineering Treatment And Reuse. Metcalg and Eddy. s.1. : Mc Graw.

Water Resources Engineering. Tchobanoglous, G. 1985. 1985.

\section{Biography}

Ridi Nurardiansyah is the name of the author of this journal. The author was born in Tangerang on February 24 , 1997. The writer studied at SDN Balaraja 3 (graduated in 2009), went on to Setu Bekasi 4 Junior High School (graduated in 2012), and Bekasi MM2100 Bekasi Industrial Vocational School (graduated in 2015), and the University Mercu Buana was finally able to take a period of study at the Faculty of Civil Engineering. 